\title{
FIXED POINTS OF SEQUENCES OF LOCALLY EXPANSIVE MAPS
}

\author{
HARVEY ROSEN
}

\begin{abstract}
A stability theorem for fixed points of a uniformly convergent sequence of open, locally expansive maps is obtained.
\end{abstract}

1. Introduction. When does a sequence of fixed points $a_{n}$ of a convergent sequence of functions $f_{n}$ from a locally compact space $X$ into itself converge to a fixed point $a_{0}$ of the limit, $f_{0}$ ? In [3], Fraser and Nadler show it does if the functions are contractive and the convergence is pointwise. Here, we show it does if all the functions are open, locally expansive on a compact connected absolute neighborhood retract $X$ and the convergence is uniform. The idea is easy enough. We lift the sequence and its limit to the universal covering space $\tilde{X}$ of $X$ and then show the inverses of the lifts satisfy a result like Theorem 1 in [3] but for locally contractive maps. The author gratefully acknowledges a helpful communication from Professor Paul Duvall in which Theorem 22 of [1] is simplified and extended to locally expansive maps.

Let $(X, d)$ be a compact metric space. A continuous function $f: X \rightarrow X$ is called locally expansive (or locally $\varepsilon$-expansive) if there is an $\varepsilon>0$ such that $0<d(x, y)<\varepsilon$ implies $d(f(x), f(y))>d(x, y)$. Rosenholtz shows in [4] that if such a function $f$ is also open and if $X$ is also connected, then $f$ has a fixed point.

An example of a locally expansive map of the torus $S^{1} \times S^{1}$ onto itself is $f(z, w)=\left(z^{2}, w^{3}\right)$ where the unit circle $S^{1}$ is viewed as a subset of the set of complex numbers. We may think of $f$ mapping meridians twice around themselves and longitudes three times around themselves.

2. Stability of fixed points. Let $X$ be a compact connected ANR (e.g., a closed, connected $n$-manifold) with metric $d$, and let $\pi: \tilde{X} \rightarrow X$ be a covering projection. According to Theorem 1 in [1], there is a complete metric $\tilde{d}$ for $\tilde{X}$ and $\eta>0$ such that if $\tilde{d}(x, y)<\eta$, then $\tilde{d}(x, y)=d(\pi(x), \pi(y))$.

LEMMA 1. If $g_{i}: \tilde{X} \rightarrow X$ is a continuous function for $i=0,1,2, \ldots$ and if the sequence $\left\{g_{i}\right\}_{i=1}^{\infty}$ converges uniformly to $g_{0}$, then there exist lifts $\tilde{g}_{i}: \tilde{X} \rightarrow \tilde{X}$ of $g_{i}$ such that $\left\{\tilde{g}_{i}\right\}_{i=1}^{\infty}$ converges pointwise to $\tilde{g}_{0}$.

Received by the editors September 1, 1977 and, in revised form, February 22, 1978.

AMS (MOS) subject classifications (1970). Primary 54H25, 54E40, 54A20; Secondary 54E45, 54F40, 55A 10 .

Key words and phrases. Fixed points, locally expansive maps, uniformly convergent sequence, universal cover, connected ANR compacta. 
Proof. Define a continuous function $f: \tilde{X} \times\left\{0,1, \frac{1}{2}, \frac{1}{3}, \ldots\right\} \rightarrow X$ by $f(x, t)=g_{i}(x)$ if $t=1 / i$ but $f(x, t)=g_{0}(x)$ if $t=0$. Since $X$ is an ANR, there is a continuous function $F: \tilde{X} \times[0,1 / n] \rightarrow X$ for some $n$ such that $F=f$ on $\tilde{X} \times\{0,1 / n, 1 /(n+1), \ldots\}$. By two applications of the lifting theorem, [5, p. 76], there exists a lift $\tilde{g}_{0}$ of $g_{0}$ (i.e., $F(x, 0)=\pi \tilde{g}_{0}$ ), and there is a continuous function $F^{\prime}: \tilde{X} \times[0,1 / n] \rightarrow \tilde{X}$ such that $F^{\prime}(x, 0)=\tilde{g}_{0}$ and $\pi F^{\prime}=F$. Define $\tilde{g}_{i}(x)=F^{\prime}(x, 1 / i)$ on $\tilde{X}$ for $i \geqslant n$. Therefore $\tilde{g}_{i}$ is a lift of $g_{i}$ and $\left\{\tilde{g}_{i}\right\}_{i=1}^{\infty}$ converges pointwise to $\tilde{g}_{0}$.

Remarks. This lemma is false if we only assume $\left\{g_{i}\right\}_{i=1}^{\infty}$ converges pointwise to $g_{0}$. Some improvements can be made. If $g_{0}=f_{0} \pi$ and if $a_{0}$ is a fixed point of $f_{0}$, then according to the lifting theorem, we can require any $\tilde{a}_{0}$ in $\pi^{-1}\left(a_{0}\right)$ to be a fixed point of a function $\tilde{g}_{0}$ to which we lift $g_{0}$. If $f_{0}$ is open and locally expansive, then it follows from Theorem 3 in [2] that $\tilde{g}_{0}$ has only one fixed point. Since $F^{\prime}$ is uniformly continuous on each compact subset $K \times[0,1 / n],\left\{\tilde{g}_{i}\right\}_{i=1}^{\infty}$ converges uniformly to $\tilde{g}_{0}$ on each compact subset $K$ of $\tilde{X}$.

LEMMA 2. If $X$ is a compact, connected, locally connected space with metric $d$ and if $f_{i}: X \rightarrow X$ is an open locally $\varepsilon$-expansive map for $i=0,1,2, \ldots$, then there is an open cover $\left\{W_{\beta}\right\}$ of $X$ evenly covered by $f_{i}$ for $i=0,1,2, \ldots$

Proof. Let $\left\{W_{\beta}\right\}$ be an open cover of $X$ such that each $W_{\beta}$ is connected and has diameter $<\varepsilon$. Let $i$ and $\beta$ be any specific indices, and let $r$ denote the diameter of the open set $W_{\beta}$. We first show that each component $C$ of $f_{i}^{-1}\left(W_{\beta}\right)$ has diameter $<\varepsilon$. On the contrary, assume some component $C$ has diameter $\geqslant \varepsilon$. Since $C$ is connected, there are points $a$ and $b$ in $C$ such that $r<d(a, b)<\varepsilon$. Therefore $d\left(f_{i}(a), f_{i}(b)\right)>d(a, b)>r$. That is, $f_{i}(a) \notin W_{\beta}$ or $f_{i}(b) \notin W_{\beta}$, a contradiction. This shows $C$ has diameter $<\varepsilon$.

Since $f_{i}$ is a locally $\varepsilon$-expansive map and since each $C$ is an open set, $f_{i}$ is a 1-1 open function on $C$. We maintain $f_{i}$ maps each $C$ onto $W_{\beta}$. Assume otherwise. Since $W_{\beta}$ is connected, there is a point $y$ of $W_{\beta}$ in the boundary of $f_{i}(C)$. Corresponding to this $\beta$ and this $i$ is some neighborhood $N$ of $y$ in $W_{\beta}$ that is evenly covered by $f_{i}$. Since $N$ meets $f_{i}(C)$, some component $D$ of $f^{-1}(N)$ is a subset of $C$. Therefore $y \in N=f_{i}(D) \subset f_{i}(C)$, an impossibility because $y$ is in the boundary of the open set $f_{i}(C)$. We have shown that for arbitrary $i$ and $\beta, f_{i}$ maps each component of $f_{n}^{-1}\left(W_{\beta}\right)$ homeomorphically onto $W_{\beta}$; i.e., $\left\{W_{\beta}\right\}$ is evenly covered by each $f_{i}$.

THEOREM. Let $X$ be a compact connected ANR and let $f_{i}: X \rightarrow X$ be an open locally $\varepsilon$-expansive map for $i=0,1,2, \ldots$ such that the sequence $\left\{f_{i}\right\}_{i=1}^{\infty}$ converges uniformly to $f_{0}$. Then for each fixed point $a_{0}$ of $f_{0}$, there exist fixed points $a_{i}$ of $f_{i}$ such that $\left\{a_{i}\right\}_{i=1}^{\infty}$ converges to $a_{0}$.

Proof. According to Lemma 2, there exists an open cover $\left\{W_{\beta}\right\}$ of $X$ evenly covered by $f_{i}$ for $i=0,1,2, \ldots$ We may assume that each $W_{\beta}$ is 
evenly covered by $\pi$ and by each $g_{i}=f_{i} \pi$. By Lemma 1 , there exist lifts $\tilde{g}_{i}$ : $\tilde{X} \rightarrow \tilde{X}$ such that $\left\{\tilde{g}_{i}\right\}_{i=1}^{\infty}$ converges pointwise to $\tilde{g}_{0}$. We let $\tilde{a}_{0}$ in $\pi^{-1}\left(a_{0}\right)$ denote the fixed point of $\tilde{g}_{0}$. Since each $\tilde{g}_{i}$ is a covering projection, each $\tilde{g}_{i}$ is a homeomorphism, [5, Corollary 7, p. 77]. Next, we verify that there is a $\delta>0$ such that $\tilde{g}_{i}^{-1}$ is locally $\delta$-contractive if $i$ is large enough. From this it would follow that $\left\{\tilde{g}_{i}^{-1}\right\}_{i=1}^{\infty}$ converges pointwise to $\tilde{g}_{0}^{-1}$.

For each $x$ in $X, x$ is in some $W_{\beta}$. Choose a connected open neighborhood $U_{x}^{\prime}$ of $x$ in $W_{\beta}$ such that each component of $f_{0}^{-1}\left(U_{x}^{\prime}\right)$ has diameter $<\varepsilon$ and each component of $\pi^{-1}\left(U_{x}^{\prime}\right)$ and $\left(f_{0} \pi\right)^{-1}\left(U_{x}^{\prime}\right)$ has diameter $<\eta$. Let $U_{\underline{x}}$ be a connected open neighborhood of $x$ in $U_{x}^{\prime}$ such that its closure, $\bar{U}_{x}$, is contained in $U_{x}^{\prime}$. Finitely many $U_{x}$ cover $X$; call them $\left\{U_{\alpha}\right\}$. Let $\varepsilon^{\prime}=$ $\min _{\alpha}\left\{d\left(\bar{U}_{\alpha}, X-U_{\alpha}^{\prime}\right)\right\}$. Since $f_{i} \rightarrow f_{0}$ uniformly, there exists an $N$ such that for every $i>N$ and for every $x \in X, d\left(f_{i}(x), f_{0}(x)\right)<\varepsilon^{\prime}$. We show that for every $i>N$, each component of $f_{i}^{-1}\left(U_{\alpha}\right)$ has diameter $<\varepsilon$. If $x \in f_{i}^{-1}\left(U_{\alpha}\right)$, then $f_{i}(x) \in U_{\alpha}$ and since $d\left(f_{i}(x), f_{0}(x)\right)<\varepsilon^{\prime}, f_{0}(x) \in U_{\alpha}^{\prime}$. That is, $x \in$ $f_{0}^{-1}\left(U_{\alpha}^{\prime}\right)$. Therefore $f_{i}^{-1}\left(U_{\alpha}\right) \subset f_{0}^{-1}\left(U_{\alpha}^{\prime}\right)$, whose components each have diameter $<\varepsilon$. Similarly, we can show that for every $i>N$, each component of $\left(f_{i} \pi\right)^{-1}\left(U_{\alpha}\right)$ has diameter $<\eta$. Let $\delta<\eta$ be a Lebesgue number for $\left\{U_{\alpha}\right\}$. It follows that for every $i>N, \tilde{g}_{i}^{-1}$ is locally $\delta$-contractive. For, if $\tilde{d}(a, b)<\delta$, then both $\pi(a)$ and $\pi(b)$ belong to the same $U_{\alpha}$, and

$$
\begin{aligned}
\tilde{d}(a, b) & =\tilde{d}\left(\tilde{g}_{i} \tilde{g}_{i}^{-1}(a), \tilde{g}_{i} \tilde{g}_{i}^{-1}(b)\right) \\
& =d\left(\pi \tilde{g}_{i} \tilde{g}_{i}^{-1}(a), \pi \tilde{g}_{i} \tilde{g}_{i}^{-1}(b)\right)=d\left(f_{i} \pi \tilde{g}_{i}^{-1}(a), f_{i} \pi \tilde{g}_{i}^{-1}(b)\right) \\
& >d\left(\pi \tilde{g}_{i}^{-1}(a), \pi \tilde{g}_{i}^{-1}(b)\right)=\tilde{d}\left(\tilde{g}_{i}^{-1}(a), \tilde{g}_{i}^{-1}(b)\right) .
\end{aligned}
$$

Choose $r>0$ so small that $r<\delta$ and $K^{\prime}=\left\{x \in \tilde{X}: \tilde{d}\left(\tilde{a}_{0}, x\right) \leqslant r\right\}$ is compact. Since $\tilde{g}_{i} \rightarrow \tilde{g}_{0}$ uniformly on $K$, there exists an $M$ such that for all $n>M$ and for $x \in K^{\prime}$,

$$
\delta>r>\tilde{d}\left(\tilde{g}_{0}(x), \tilde{g}_{n}(x)\right)>\tilde{d}\left(\tilde{g}_{n}^{-1} \tilde{g}_{0}(x), x\right)=\tilde{d}\left(\tilde{g}_{n}^{-1} \tilde{g}_{0}(x), \tilde{g}_{0}^{-1} \tilde{g}_{0}(x)\right) .
$$

That is, $\tilde{g}_{i}^{-1} \rightarrow \tilde{g}_{0}^{-1}$ uniformly on $\tilde{g}_{0}\left(K^{\prime}\right)$. Choose $s>0$ so that $K=\{x \in \tilde{X}$ : $\left.\tilde{d}\left(\tilde{a}_{0}, x\right) \leqslant s\right\} \subset \tilde{g}_{0}\left(K^{\prime}\right)$. According to the proof of Theorem 1 in [3], for $i$ large enough, $\tilde{g}_{i}^{-1}$ maps $K$ into itself and has its unique fixed point $\tilde{a}_{i}$ in $K$. This shows $\left\{\tilde{a}_{i}\right\}_{i=1}^{\infty}$ converges to $\tilde{a}_{0}$. Let $a_{i}=\pi\left(\tilde{a}_{i}\right)$. It follows that $a_{i}$ is a fixed point of $f_{i}$, and $\left\{a_{i}\right\}_{i=1}^{\infty}$ converges to $a_{0}$.

\section{REFERENCES}

1. P. F. Duvall, Jr. and L. S. Husch, Analysis on topological manifolds, Fund. Math. 77 (1972), 75-90.

2. M. Edelstein, On fixed and periodic points under contractive mappings, J. London Math. Soc. 37 (1962), 74-79.

3. R. B. Fraser, Jr. and Sam B. Nadler, Jr., Sequences of contractive maps and fixed points, Pacific J. Math. 31 (1969), 659-667. 
4. Ira Rosenholtz, Evidence of a conspiracy among fixed point theorems, Proc. Amer. Math. Soc. 53 (1975), 213-218.

5. E. H. Spanier, Algebraic topology, McGraw-Hill, New York, 1966.

Department of Mathimatics, University of TeXas at Austnn, Austan, TeXas 78712

Departmant of Mathematics, Universtity of Alabama, University, Alabama 35486 (Current address) 